\title{
回应失恋大学生网络心理咨询 On the Rhetorical Strategies 的话语修辞策略研究 of Psychological E-Counselling Discourse in Response to Lovelorn College Students
}

\section{毛浩然*}

华侨大学外国语学院

泉州 福建 中国, 362021

张 芳

华侨大学图书馆

泉州 福建 中国, 362021 125479358@qq.com

im02@163.com
Mao Haoran

Huaqiao University

School of Foreign Languages Quanzhou, Fujian, China 362021

Zhang Fang

Huaqiao University Library

Quanzhou, Fujian, China 362021
本研究基于劝导失恋大学生的成功案例, 剖析网络心理咨询的优势和话语修辞策略。研 究发现: (1) 大学生网络心理咨询可分为 Email 延时话语和在线 QQ 即时话语。远程便捷性、 匿名私密性、资质安全性, 是失恋大学生日益 倾向于向自己信任的教师寻求网络心理咨询帮 助的主要原因, 而这正是传统面询并不具备的 优势。网络心理咨询与时俱进, 具有广阔的应 用前景; (2) 回应失恋大学生网络心理咨询的 话语修辞策略主要有: A) 巧设环环相扣的引导 性问题，了解失恋学生的真实困扰盲点; B) 运 用切中要害的适时反问, 切断失恋学生的消极
This paper analyses the advantages and rhetorical strategies of psychological E-Counselling discourse, based on successful cases in response to lovelorn college students. It is found that: (1) Psychological E-Counselling discourse of college students can be divided into time-lapse discourse via email and impromptu online discourse via QQ. Remote communication via the internet, privacy by anonymity, and trust in teachers as consultants are the three main reasons for the increasing number of lovelorn college students asking their teachers for psychological E-Counselling, which has advantages compared to traditional face-to-face counselling, and also broad application prospects;

* [ 作者简介] 毛浩然, 博士, 华侨大学外国语学院教授, 博士生导师。研究方向: 话语修辞和二语习得。 张芳, 硕士, 华侨大学图书馆副研究馆员。研究方向: 信息管理和教育心理。

[基金项目] 本文系教育部人文社会科学研究规划青年基金项目 “外语教师评课话语实景认知研究” (编 号: 11YJC740075）系列成果之一。 
回应失恋大学生网络心理咨询 96
On the Rhetorical Strategies of Psychological Discourse in

E-Counselling Response to Lovelorn College Students
话轮循环；C）建立得当的心理参照系，弱化失 恋学生极端悲观的自我判断; D) 引用切题应景 的哲理故事警言潮语, 引发失恋学生的反省与 共鸣；E）提供重启心灵的具体化建议，引导失 恋学生投身建设性事务; F) 借力互动平台群体 话语，强化多管齐下的劝导实效。

[关键词] 失恋; 大学生; 话语修辞; 网络心理 咨询

\section{1. 引言}

先举一实例说明心理咨询并非高深莫测: 有位家长找到某大学心理系 $\mathrm{C}$ 教授，说自己的儿子左手不灵便，因未能及时找到最好的医生帮其治疗，就一 直后悔愧疚导致严重失眠, 寢食难安。C 教授是这样开导他的: “周恩来的手 臂在延安时不慎落马摔断，本来应该去前苏联治疗，但当时战事正紧，无法脱 身，所以未能及时得到最好的救护，你看周总理的鼓掌姿势都是比较特别的， 就是这个原因。但是否影响其后期工作甚至影响其成为一代伟人呢? 没有, 对 吧？他是整条手臂啊，你儿子还只是一只手不太灵便，又有什么太大的关系 呢? ”这位家长顿时心中释然。这次心理咨询之所以奏效, 主要是心理参照系 在起作用，其潜台词是：“你看，比你更厉害更显赫的人，他的境遇比你儿子 还惨呢，他都没事，那你还有什么好担心的呢？”当然，深究起来，心理咨询 师的话语有时确实也有 “站着说话不腰疼”之嫌，特别是当自己遇上类似麻烦 时可能就失效了 ${ }^{1}$, 但这并不影响其在接受他人心理咨询时所产生的积极效果。

在校大学生谈恋爱已是常态, 失恋并非个案, 但不少大学生自我心理调适 能力不强，谙于享受爱情交织的甜蜜却疏于承受爱情沉没的成本，尤其是重度 失恋者在应激状态下容易因玻璃心而走极端, 甚至厌世轻生。调查表明, 在这 种危急关头, 极少数失恋大学生求助于心理咨询师, 而更多倾向于通过网络渠 道向自己信任的老师寻求心理咨询帮助。网络心理咨询话语具有鲜明的特点,

过了一段时间, C 教授的儿子的一根手指不慎受伤, 经治疗后一直处于弯曲状态无法回直, C 教授就 一直纠结难受, 别人问他: “你不是说周恩来都没事嘛?” 他说: “劝别人可以, 自己遇上了, 就不一定 见效了，很少有感同身受这回事，除非你真的经历过。” 
如即时性、延时性、匿名性、时空自由性等, 其互动语言既保持心理咨询的语 言特点, 又凸显网络语言的特性。大学生网络心理咨询主要可分为 Email 延时 话语和在线 QQ 即时话语。Email 延时话语通常被失恋轻伤程度的大学生所采 用, 在访谈中, 学生也表示 TA 们只是抱着 “试试看, 有回复最好, 没回复拉 倒” 的心理, 并不奢望老师真的能有空并用心回复。而在线 QQ 即时话语则被 失恋轻伤和重伤程度的大学生所通用, 一般留言后都会希望老师能及时回应。

调查结果表明: 远程便捷性、匿名私密性、资质安全性，是失恋大学生日 益倾向于向自己尊敬的教师寻求网络心理咨询帮助的主要原因,而这正是传统 面询所欠缺的。同时, 访谈中发现, 向自己的老师寻求网络心理咨询帮助, 多 为公益免费型, 这也是网络心理咨询颇受大学生欢迎的一个因素。但更重要的 是安全感, 访谈中的大学生们表示, 人在激奋心理状态下, 安全感和求助欲占 主导地位, 一般在不太严重时才会考虑免费公益的因素, 如果很严重, 则经济 因素就不在考虑之列。调查结果还显示: 失恋状态下, 私密性、安全性的缺乏 以及在咨询期间担心被发现等, 是失恋大学生对网络心理咨询的主要担忧。总 而言之, 网络心理咨询与时俱进, 具有广阔的应用前景, 尤其是在大学生失恋 领域, 向自己信任的老师寻求心理咨询帮助, 因其即时性、私密性、便捷性、 安全性和有效性而逐渐为大学生所青睐。本研究基于劝导失恋大学生的成功案 例, 旨在剖析网络心理咨询话语的优势和修辞策略, 提供一系列建设性的操作 建议和话语修辞策略, 帮助教育工作者为身处类似情境的学生提供专业有效的 心理咨询。

\section{2. 核心概念定义}

\section{1 心理咨询与网络心理咨询}

心理咨询是指来询者就心理、精神方面存在的问题, 向咨询人员进行述说、 询问和商讨, 以求解决问题及平衡心态的过程, 是心理咨询师运用心理学专门 的理论和技巧, 在良好的人际关系氛围中, 启发和帮助来询者, 挖掘其潜能, 从而找到产生心理问题的原因, 辨明心理问题的性质, 寻求摆脱心理困扰的条 件和对策, 达到恢复心理平衡、提高适应能力、最终增进身心健康的活动（张 芳、毛浩然，2008：78-81）。

John M. Grohol（1995）最早将网络心理治疗（e-therapy）定义为一种 新型的帮助人们解决生活和工作问题的咨询治疗模式。美国国家注册心理咨询 师协会（National Board of Certified Counsellors, NBCC）把网络心理咨询”定 义为 “心理咨询师与当事人使用电子邮件、聊天室或网络视频设备进行远距离

\footnotetext{
2 网络心理咨询的英译术语有多种, 如: Online Counseling, Cyber Counseling, Online-Therapy 和 Internet Counseling (McCrickard \& Buttler, 2005; Cook \& Doyle, 2002; Pollock, 2006; Tanrikulu, 2009)。 本研究更倾向于将其译为 “E-Counselling”。
} 
回应失恋大学生网络心理咨询 的话语修辞策略研究
On the Rhetorical Strategies of Psychological Discourse in

E-Counselling Response to Lovelorn College Students

的同步实时或异步非实时的互动”（NBCC，2001）。 ${ }^{3}$

大学生网络心理咨询指高校心理咨询工作者借助互联网这一媒介, 运用心 理学的原理和方法, 通过远距离即时或非即时互动, 对在心理适应方面出现问 题并企求解决的在校大学生给予心理层面的指导和帮助的过程 (邱芬, 2008)。 大学生网络心理咨询的种类很多, 目前应用比较广泛的有电子邮件（Email） 咨询、QQ 咨询、在线聊天室和其他在线互动平台咨询。

\section{2 心理咨询、心理治疗和心理辅导的差别}

心理咨询、心理治疗和心理辅导均具有三个目的：预防（prevention）、 干预（intervention）和矫正（remedy）。心理治疗（psychotherapy）是对 有心理疾病的人进行的以改正其行为、情感和想法为目的的心理咨询过程; 心 理辅导是针对学生群体进行的以促进其身心健康发展为目标的心理咨询过程; 心理咨询则填补了这两者之间的空白地带, 服务于那些既不属于学校系统, 又 不是被诊断为有心理疾病的人群。心理咨询与心理治疗最大的区别在于服务的 对象和面对的问题不同, 区别的关键在于求助者是否具有心理疾病。例如: 情 绪低落或感觉郁闷与抑郁症并非一回事, 后者需要通过心理测量、评估和诊断 才能被确定，而我们却都可以声称自己今天很郁闷。在方法和具体操作上，心 理治疗更倾向于使用医学模式。心理咨询虽然也遵循发现问题、分析问题和解 决问题的思路，但在方法上可以灵活多样，不拘一格。除此之外，心理咨询还 有一个积极意义, 即强调预防性。心理咨询和心理辅导最大的差别在于工作的 对象和地点。心理辅导的工作地点是学校, 面对的是全体学生。因此, 心理辅 导从发展的角度出发, 重在文化了解和适应性教育, 其核心内容是学习、社交 与情感发展和职业发展。

\section{3. 研究起点界定}

网络心理咨询已惠及很多领域, 例如在婚姻家庭（Pollock，2006）、戒 烟（Mallen, Blalock \& Cinciripini, 2006) 、抑郁症 (Christensen, Griffiths, \& Jorm，2004）和焦虑紊乱 (Kenardy, McCafferty \& Rosa，2003) 等方面的 心理咨询。下面简要梳理一下网络心理咨询先行研究的主要成果和研究不足。

\section{1 心理咨询的主要学术流派和研究现状}

对心理咨询和心理治疗影响较大的有四个学术流派: 精神分析心理学、行 为主义心理学、人本主义心理学和认知心理学。弗洛伊德提出了精神创伤是引

\footnotetext{
3 英语原文: The continuously growing practice of online counseling, which is defined as "asynchronous and synchronous distance interaction among counselors and clients using e-mail, chat, and video conferencing features of the Internet to communicate."
} 
起精神疾病的主要原因, 主张用精神分析方法来发掘病人被压抑在潜意识内的 心理矛盾以治好病人，从而突破了过去纯粹靠医药、手术和物理方法的生物医 学模式的束缚, 开创了一条重视心理治疗的现代医学模式的新途径。其主要理 论基础包括潜意识与压抑理论、人格结构说。行为主义创始人华生认为人的行 为都是对环境刺激所作出的反应。新行为主义者斯金纳认为人的行为主要是由 操作性条件反射所构成。行为疗法是根据巴甫洛夫的经典性条件反射和斯金纳 的操作性条件反射原理衍生出来的心理治疗技术, 但行为疗法的理论着眼于行 为本身, 忽视了人的心理因素, 如认知、情感和意志的作用。矫正行为必须同 时注意消除心理上的障碍。

人本主义重视人的价值与尊严，研究的中心是基本的人性（自我的成长、 需要、天赋潜能、自我实现等）。其代表人物马斯洛和罗杰斯的基本理论是需 要层次论和自我实现论。其疗法强调咨询师与咨询者的交谈是在咨询者自主的 情况下进行的, 不受任何拘束。罗杰斯还对这种方法提出了几个必要条件, 如 治疗者必须与患者建立良好的人际关系; 治疗者必须以真诚的态度对待患者等, 这些条件与人本主义心理学所强调的尊重人的价值与尊严的精神相一致。认知 心理学强调人的行为是受人的认知所支配的。心理咨询的关键是指导病人改变 原来的认知结构而确立新的认知结构。认知学派的 “情绪 $\mathrm{ABC}$ 理论” 认为: 人 的情绪反应不是由外界诱发性事件（activating event）引起, 而是由人对事 件的认知和信念（beliefs）所引起。其 RET 心理治疗过程共分四个阶段：心 理诊断阶段、领悟阶段、沟通阶段和再教育阶段。

精神分析心理学、行为主义心理学、人本主义心理学和认知心理学各有千 秋, 需因人而异辩证施治, 简而言之, 治无定法, 治有常法, 贵在得法。心理 咨询的研究重点在于注重心理咨询的过程和效果研究, 考察咨询过程变量对咨 询效果的影响。心理咨询方式包括直接咨询和间接咨询, 个别咨询和团体咨询。 按咨询途径可分为电话咨询、通信咨询、门诊咨询、现场咨询、报刊专栏咨询 等。本研究重点关注大学生网络在线心理咨询的话语修辞策略研究。

\section{2 大学生心理咨询的研究现状与不足}

在科学网 (web of science) 上, 我们以 “psychological counselling” 和 “university student” 为关键词进行检索 (以 college student 为主题词检索的 结果类似），显示出以下研究趋势： 
回应失恋大学生网络心理咨询 的话语修辞策略研究
On the Rhetorical Strategies of Psychological Discourse in E-Counselling Response to Lovelorn College Students

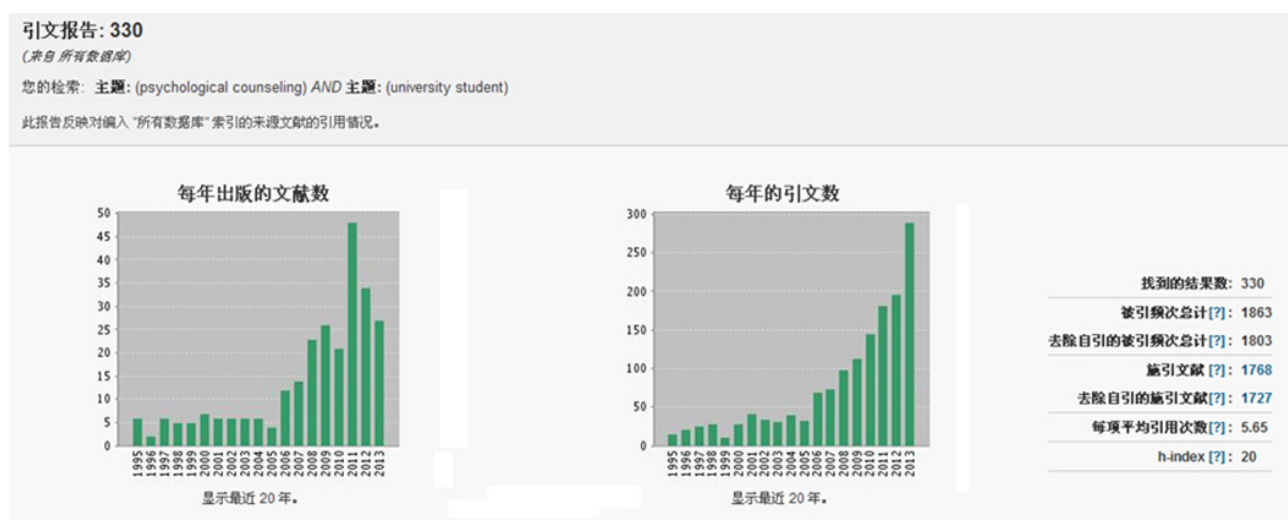

图 12005 年以来的大学生心理咨询文献数和引文数

如图 1 所示, 从 2006 年起, 大学生心理咨询研究成果量和每年引文数量 开始急速上升，成为全球性的热点之一。特别是 2011 2013 间，其发文数量 和引文数量达到近年来的顶峰。从发文数量和引文数量来看, 大学生心理咨询 研究的发展经历了三个阶段： 20 世纪 60 70 年代初为萌芽阶段； 20 世纪 70 年代初 21 世纪初为重视阶段; 21 世纪初至今为急速发展阶段。进入 21 世纪 以后, 大学生心理咨询成为学者们研究的焦点, 大学生心理咨询也空前发展, 说明大学生心理健康问题已成为各高校突出的普遍性问题, 引起包括学术界在 内的全社会的关注。

从研究内容来看, 大学生心理咨询研究包括心理健康 (mental health), 行为科学 (behavioural science) 、学校心理学 (school psychology)、保健 (health care)、饮食紊乱（eating disorder）、艾滋病（HIV）以及生理行为 (physical activity) 等 20 个方面。其中心理健康的咨询研究成果量最多, 为 517 个, 由此可见大学生心理健康是最受关注的问题, 而行为科学、校园心理、 身体健康、HIV、社会支持、性泛滥和体育活动等, 也是大学生心理咨询研究 的主要问题。

我国大学生心理咨询研究起步较晚, 开始于上世纪八十年代中期, 到 90 年代中期得到重视, 真正蓬勃发展则是在 2008 年之后。张芳、毛浩然 (2008: 79-80）提出, 大学生心理咨询的实用方法可归结为以下几种：聆听法、疏泄 法、激励法、个体咨询与团体辅导相结合法、自我心理保健法。另有学者如晏 小萍（2008：63-71）对心理咨询话语结构进行了研究，通过对心理咨询中谈 话结构微观层面的语言结构分析, 指出谈话参与者的话语权势在职业场景的谈 话活动中有其预设的规律。谈话者在谈话活动中享有的话语权大小受到话语角 色的制约。心理咨询谈话结构的分析结果显示, 话语权在谈话中呈动态形式, 其话语置控权和话语赋权行为可以通过话语结构布局和谈话人担当的话语角 色得以实施。宋大伟（2008：221-223）对网络心理咨询局限性和应用对策的 研究, 提出网络心理咨询的局限性大致可归结为: 途径和方式的局限性、治疗 
环境的局限性、咨询渠道的局限性和使用工具的局限性。

总体而言, 虽然近年来我国大学生心理咨询研究发展迅速, 但和国外相比, 还有很大的发展空间。当前我国大学生心理咨询的不足之处主要有: 开展心理 咨询的高校数量不均衡; 开展心理咨询的高校心理咨询师数量不够、心理咨询 质量参差不齐; 心理咨询室形同虚设现象严重; 大学生心理咨询的理论与实践 研究仍有较大差距; 心理咨询话语研究严重滞后, 等等。

\section{3 大学生网络心理咨询的研究现状与不足}

进入 21 世纪后, 网络渗入社会生活的方方面面, 网络心理咨询的成果量 和引文数量空前增长。网络为心理咨询发展提供了一个很好的工具和平台, 网 络心理咨询与时俱进, 具有广阔的应用前景。

网络心理咨询研究主要有以下几个方面：网络心理咨询的特点、对象、过 程、服务、形式、理论、技术、伦理、效果、语言、话语分析、政策、监管和 推广等方面。我们以 “psychological counselling” 和 “internet” 为关键词 在 web of science 数据库上进行搜索, 其所涉内容前 6 位分别为: internet (互联网), mental health (心理健康), child (儿童), breast cancer (乳 腺癌）, physical activity（生理行为）和 health care（保健）。

国外网络心理咨询的相关研究包括两个方面：（1）学生求助于网络、通 讯工具和媒体交流等途径的原因、动机、态度、优势和性别差异等, 如 K.J. Glasheen 等 (2015) 研究了性别差异、先前咨询经验、学生年级以及心理压 力程度对学生寻求网络咨询动机的影响, 并探讨了学生在网络咨询中较常讨论 的话题。Johansson 等 (2007)、Smith (2012) 和 Maclean 等 (2013) 等的 研究指出了男生寻求心理咨询的概率比女生低。（2）Kate Dunn（2012）等 研究了网络咨询的过程、结果以及效度等, Robert L. Glueckauf（1993）等比 较了以视频和面询两种不同方式进行家庭心理咨询的效果, 发现两种治疗方式 均有效, 治疗方式并不影响治疗效果, 这说明通过网络将有问题或存在危机的 家庭, 从偏远的地方连接到治疗环境下进行网络治疗是有效的。那么有其他问 题的群体 (如: 大学生) 通过网络与有类似问题的人群沟通, 也很可能从中获 益。网络心理咨询和传统面询的比较研究说明网络咨询是有效的, 具有广泛的 应用前景。但正如 Edwards-Hart \& Chester（2010）所指出的，尽管年轻大 学生倾向于在网上查询健康信息, 但是辨别或识别这些信息的内容、本质以及 其传递方式的研究却较少。

总体而言, 大学生网络心理咨询研究的不足在于: 其一, 大部分研究仅从 宏观角度分析大学生网络心理咨询的问题、对策及效果, 少有针对特定类别(如 恋爱) 具体案例进行的实证研究; 其二, 大部分研究从网络咨询本身的问题出 发, 很少从话语修辞的角度分析咨询人和被咨询人的话语模式、修辞策略等。 
回应失恋大学生网络心理咨询 的话语修辞策略研究
On the Rhetorical Strategies of Psychological Discourse in

E-Counselling Response to Lovelorn College Students

\section{4. 回应大学生网络心理咨询的话语修辞策略}

助人于患难之时, 雪中送炭远比锦上添花更具真情, 雪中送炭并非只指赠 予财物。为受若的人说一句解围的话, 为沮丧的人说一句鼓励的话, 为疑惑的 人说一句提醒的话, 为痛苦的人说一句安慰的话, 看似小小的帮助, 也许就能 助人重新焕发生命活力, 甚至是救人一命。日常生活中, 人们或多或少都有过 心理咨询的实践，并非只有心理咨询师才有涉猎，师生间 “无证公益”式的“业 务量”在实践中的比例更是不小。笔者近三年来就成功劝和 9 对闹离婚的夫妇, 挽救 6 位重度失恋欲轻生的学生。值得一提的是, 部分持有国家心理咨询师证 书者少有实践操作, 偶尔为之, 可能收效甚微, 且容易出现消极的心理共振, 影响自身的心理健康。因此, 有兴趣兼顾学生心理咨询的教师, 需具备较强的 自我心理调适能力（尤其是心理自洁能力）, 方可为之。同时, 最好要多学习 一些心理咨询的专业知识。如果能获得国家心理咨询师证书, 当然就更好了。

笔者对心理咨询的通俗理解可分解为: $50 \%$ 是为咨询者建立心理参照系 (退 一步海阔天空）、25\%倾听咨询者的倾诉（当好垃圾桶，有什么不开心的事尽 管说）和 $25 \%$ 建设性对策方案（接地气、具体化、可操作）。少数情况则是 7 $0 \%$ 为咨询者建立心理参照系（退一步海阔天空）和 30\%倾听咨询者的倾诉，这 种情况只需要心理调适而不需要给出建设性对策方案。

本研究主要为案例分析法,语料来源为平时积累的失恋大学生网络心理咨 询实践的典型成功案例实录 (以下邮件和 $\mathrm{QQ}$ 互动内容作为研究语料已取得学 生当事人同意），并在语料分类分析的基础上辅以调查与访谈。

\section{1 Email 类心理咨询实例分析}

亲爱的毛老师:

您好! 为了不占用您太多时间, 学生我直接开门见山啦。我最近失恋疗伤 中, 很是难受, 说实话, 我的颜值比较有优势, 但在恋爱中却似乎反而变得没 优势。很想请教您一个问题，一个优雅气质的女人必须具备怎样的条件，必须 具备怎样的人生观、价值观、婚姻观，才有可能在恋爱甚至婚姻中多走一些坦 途少受一点伤害? 我知道这是个既简单又复杂的话题, 但是我相信您一定能给 我很好的建议, 让迷途中的我尽快找到方向。如果可以, 希望你能介绍几本相 关的书籍给我。很感谢您在短暂培训课堂中为大家带来简短而深刻的一堂课, 您幽默的话语令同学们开怀大笑,那一刻我们学到的已不止于关乎考试的信息, 更多的是蕴藏在您言语间的哲理智慧和心灵启迪。感谢您，期待您的回信。

祝您万事如意，桃李满天下!

汉语言文学专业学生 $\times \times \times$

显然, 这位学生属于失恋轻伤类型, 她写这封邮件主要目的不是倾诉, 而 是想吃一堑长一智, 为后续的恋爱和婚姻少走弯路而未雨绸缪, 这样的咨询, 回应时有三个重点：一是言简意赅，二是切中要害，三是具备信息差。 
笔者的 Email 回复:

$\times \times \times$ 同学:

你提的这个问题好大, 估计十部专著都不够写, 不过类似的问题确实有学 生问过我。大学生失恋原因虽然各不相同, 不过我想大道至简万物一理, 相信 你能触类旁通。下面是我当时的回答，供你参考。

女生看得见的风姿是以看不见的涵养为基础的，没有 knowledgeable，就 不太可能会有真正意义上的 decent 和 graceful。要做有智慧的美女，不要让 自己仅仅只是花瓶。妩媚最好是一种可收可放的本领, 而不要让它成为你的特 质。天生丽质是资本，经营不好就会让自己破产。

至于人生观、价值观和婚姻观，It's a long story. 简而言之，就是经济基 础与上层建筑的比例问题，智者见智，仁者见仁，得失利弊，存乎一心。有两 点是最重要的, 一是要真正学会付出与奉献, 即使得不到回报或与付出相当的 回报，也能自我开导想得开。二是未来一定要有一份属于自己的工作，即拥有 可以独挡一面的经济基础。工作的意义不仅仅是谋生，对于女性，没有经济上 的独立, 就不可能有真正意义上的人格独立。如果男朋友或未来的老公很富裕, 充其量只能让他的经济实力为你锦上添花而不能是雪中送炭。

至于看什么书，你是汉语言文学专业的，我就不班门弄斧了，只有一个原 则, 只要是能完善知识结构或陶冶情操的好书, 都值得一读, 不拘一格。但开 卷未必有益, 差的书就像地沟油, 还是不读为妙。

祝心想事成!

这封 Email 回复，第一段先以 “不过类似的问题确实有学生问过我” 来建 立学生的心理参照系, 即: 失恋并非个案, 也不是你最倒霉。“大学生失恋原 因虽然各不相同，不过我想大道至简万物一理，相信你能触类旁通。”这句话 是为了增强下文的普适性, 希望这位学生能举一反三, 而非简单的就事论事。

在第二段中我们看到, “女生看得见的风姿是以看不见的涵养为基础的, 没有 knowledgeable, 就不太可能会有真正意义上的 decent 和 graceful。要 做有智慧的美女, 不要让自己仅仅只是花瓶。妩媚最好是一种可收可放的本领, 而不要让它成为你的特质。天生丽质是资本，经营不好就会让自己破产。”这 段话切题应景, 富含哲理警言潮语, 且具备较强的信息差和启发性, 可有效引 发失恋学生的反省与共鸣, 其建设性建议的目标就是 “要做有智慧的美女”, 间接回应这位学生“我的颜值比较有优势, 但在恋爱中却似乎反而变得没优势” 的困惑。

第三段回应人生观、价值观和婚姻观的困惑。“简而言之, 就是经济基础 与上层建筑的比例问题” , 这个回应非常言简意赅, 蕴含了浓厚的学术味道, 却深入浅出, 对熟悉政治经济学基本原理的大学生来说, 理解起来驾轻就熟。 随后给出了两点建设性建议: “一是要真正学会付出与奉献, ……是未来一 定要有你自己一份属于自己的工作。” 同时强调 “对于女性, 没有经济上的独 
回应失恋大学生网络心理咨询 的话语修辞策略研究
On the Rhetorical Strategies of Psychological Discourse in

E-Counselling Response to Lovelorn College Students

立, 就不可能有真正意义上的人格独立。……只能让他的经济实力为你锦上添 花而不能是雪中送炭。”这些都为学生提供了重启心灵的具体化建议和务实的 哲理思考。

第四段简要回应, 引导失恋学生投身建设性事务, 并注重正确读书理念的 引导, 即: 好书要多读, 差书别乱读, 因为开卷未必有益。

可以看出, 这种 Email 回复更像是师生谈心与个别辅导, 适用于处于失恋 轻伤状态的大学生, 或基本痊愈需进一步巩固疗效的失恋大学生。

\section{2 在线 $\mathrm{QQ}$ 类心理咨询实例和话语修辞策略}

如下是笔者与一位 2012 级失恋新生的 $\mathrm{QQ}$ 对话实录。“终是迷惘...” 是 听过笔者讲座的一位大一新生, Labour 是笔者的 $\mathrm{QQ}$ 呢称。为了便于分析, 全程 QQ 对话语料分为三个片断。

\subsection{1 策略一: 巧设环环相扣的引导性问题, 了解真实的困扰盲点}

片断一:

终是迷惆 $\cdots 111 ; 03 ; 50$

毛老师，我最近情绪很不稳定，您有空陪我聊聊吗?

Labour 11; 05;21

你是哪位?

终是迷惆 $\cdots 111 ; 06 ; 11$

外院的, 但不是 5 班的学生, 听过您的讲座

Labour 11; 06; 45

2012 级吗? 请说!

终是迷惆 $\cdots 111 ; 06 ; 46$

恩! 我和男朋友分手了, 他说他受不了异地恋。我也好想潚 酒地离开, 可是真的好难受。我们认识了 5 年, 一开始是以 知已的身份相处的。

Labour 11；12；44

你现在是如何想如何分析的?

终是迷惆 $\cdots 11$ 1；14；47

之前他话变少了, 我问他为什么, 他说他们学院的心理测试 总分两分, 他 1.6 。他现在对周边的人都很沉默。然后不久 后就提分手，可是他说的那个理由我接受不了。

我比较内向, 从来不轻易相信别人, 可是我相信了他。因为 小时候是寄养在别人家里, 回家后跟家里其实不是很亲。 
Labour $11 ; 18 ; 43$

他如果给你一个你能接受的理由, 你就释然了吗?

终是迷恫 $\cdots 111 ; 18 ; 47$

我没把握

Labour $11 ; 20 ; 00$

其实你不是因为他的什么理由, 而是分手这个事实!

终是迷惆 $\cdots 111 ; 20 ； 29$

那我该怎么办? 我现在早上很早就醒了, 睡不着。食量变得

好小。一想到他就忍不住想哭。昨天胸口特别难受, 跟当初

高三压力过大一样。

Labour $11 ; 25 ; 52$

你是因为真的爱他, 还是因为已经付出很多, 分手心有不 甘?

终是迷惆 $\cdots 111 ; 27 ; 05$

真的爱他!

Labour 11；27；56

在什么样的情况下，你会放弃爱他?

终是迷惆 $\cdots 111 ; 28 ; 05$

我不知道

Labour 11；29；14

你好好想想, 以局外人的角度多列举几种可能, 一- 列举 出来给我看看。

终是迷惆 $\cdots 111 ; 33 ; 20$

恩! 当我的面和别的女生牵手，或当面狠狠地批我的缺点， 甚至是骂我!

我觉得自己活不长了!

Labour 11；48；26

那你觉得他现在提出分手最可能的原因是什么?

终是迷惆 $\cdots 111 ; 50 ; 31$

淢了, 如果真的是因为受不了异地恋, 证明他并没有真的把 我放在心上

终是迷惆 $\cdots 111 ; 52 ； 47$

我问过他，既然如此，为什么还要表白?（在高考结束的 那天）他说他没有想到会在不同地方读书，他说他违背填写

志愿的原则，把 $\times \times$ 学院填在 $\times \times$ 师院前面 


\section{Labour $11 ; 53 ; 40$}

现在他在哪读?

终是迷惆 $\cdots 111 ; 53 ; 53$

$\times \times$ 师院。在刚知道被 $\times \times$ 师院录取的时候他哭了。但他说 他不会放手，因为我和别的女孩不一样，他说我善于倾听, 和我在一起很自在。我从来不要他买什么。他知道我只在乎 他在身边, 而不是去哪里玩。

\section{Labour 11; 57; 57}

你是 $\times$ 州人? 他呢?

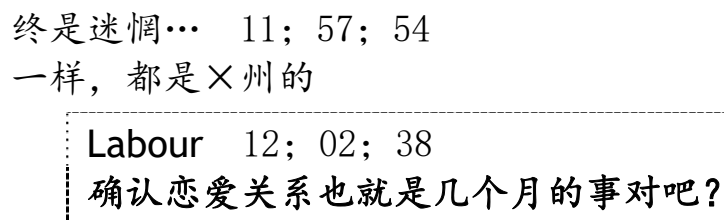

Labour 12;02;38

确认恋爱关系也就是几个月的事对吧?

\section{终是迷惆 $\cdots 12$ 12；02；43}

高考结束那天

Labour 12；03；30

其实你算是好的了

终是迷惆 $\cdots 12$ 12；03；32

什么意思?

先来看看这段对话中老师共提问了哪几类问题，使用了哪些隐性问句:

一是个人基础信息类，如：“2012 级吗?” “现在他在哪读?” “你是 ×州人？他呢？”“确认恋爱关系也就是几个月的事对吧？”

二是层层深入引导类，如： “你现在是如何想如何分析的？” “在什么样 的情况下, 你会放弃爱他? “那你觉得他现在提出分手最可能的原因是什么? ”

三是一针见血反问类，如: “他如果给你一个你能接受的理由，你就释然 了吗？” “你是因为真的爱他, 还是因为已经付出很多, 分手心有不甘? ”

四是以答代问猛药类, 如: “其实你不是因为他的什么理由, 而是分手这 个事实！”

五是祈使代替问句类，如： “你好好想想，以局外人的角度多列举几种可 能, 一一列举出来给我看看。”

当遇上学生寻求心理咨询帮助时, 千万别在未了解情况前就着急提供一系 列万金油式的建议, 那样就是无的放矢了。一定要设法让学生开口, 引导学生 生理层面的开口能促成学生心理层面的打开心扉。如果咨询者不愿意开口表达, 就用合适的问题引导其开口表达, 甚至可以先从引导咨询者点头、判断式的单 
字回应（以引导其做出肯定答复为主）, 到短语和短句回应, 再到比较完整的 表述。总之, 循序渐进地引导学生开口表达, 是心理咨询极其重要的第一步。 哪怕老师已经知道自己所问的问题学生会如何回答, 在接受心理咨询时, 依然 要为学生巧设环环相扣的引导性问题。这主要有三大好处: 一是有助于了解失 恋学生的真实困扰盲点, 不会想当然; 二是有助于打开失恋学生出于自我保护 而紧闭的心扉, 助其吐露实情; 三是有助于打开学生消极情绪的渲泄出口和通 道。本例中这位学生属于比较愿意表达的类型, 所以就干脆让她自己先多说说 情况。上文中的五类问题, 即: 个人基础信息类、层层深入引导类、一针见血 反问类、以答代问猛药类和祈使代替问句类，都是可以推而广之的设问类别。

\subsection{2 策略二: 运用切中要害的适时反问, 切断消极话轮循环}

当学生一直纠结于 “可是他说的那个理由我接受不了” 和 “既然如此, 为 什么还要表白” 时, 如果不及时切断其话轮, 将形成后续消极话轮没完没了的 死循环, 劝导者也难以掌控情绪引导的主动权。但如果直接告诉失恋学生这样 不对, 可能学生易产生抵触情绪。此时, 切中要害的适时反问, 可以四两拨千 斤。“他如果给你一个你能接受的理由, 你就释然了吗? ” “你是因为真的爱 他, 还是因为已经付出很多, 分手心有不甘? ” 这两个问题即是典型例子。

片断二:

Labour 12; $02 ; 38$

确认恋爱关系也就是几个月的事对吧?

终是迷惆 $\cdots 12 １ 2 ０ 2 ； 43$

高考结束那天

Labour 12; 03；30

其实你算是好的了

终是迷惆 $\cdots 12 １ 2 ０ 3 ； 32$

什么意思?

Labour 12；04；30

我有几个学生都是正式谈了两年以上才分手了，而且有的 都已经同居过了。

终是迷惆 $\cdots 112 ； 05 ； 42$

也许这么比我确实算是挺好的，可是…

Labour 12；07；27

他可能是真的另有想法或另有新欢, 但不想刺激你, 你也不 用去求证, 好聚好散, 两人相爱过, 不成情侣, 至少不能成 为敌人, 这是我一向的主张。 
回应失恋大学生网络心理咨询 的话语修辞策略研究
On the Rhetorical Strategies of Psychological Discourse in

E-Counselling Response to Lovelorn College Students

我们都曾安慰过别人, 也被别人安慰过, 一定有过这样的体验：安慰者说 的那些道理（比如要看开点啊、来日方长啊之类的）你都懂, 但你自己说服不 了自己, 得听别人说了, 才句句入心。为什么大多数情况下你听到的道理其实 都懂, 但仍然需要别人来说呢? 为什么有时咨询师或好友给出的不具有信息差 的对策方案也同样能对你产生积极效应呢? 这就是心理咨询很重要的一个功 能, 即: 通过倾听, 架构咨询者的负能量释放出口和通道, 并在其心理脆弱的 特殊阶段以正能量对其进行扶持强化。特别是一些容易灯下黑的盲点, 需要借 力使力, 反问质疑, 一语点醒梦中人, 从而避免失恋学生进入纠结的死循环中。

在另一例劝导大四失恋学生的对话中, 笔者叮嘱失恋学生去完成四件事, 其中前两件是均是基于切断失恋学生消极情绪循环的考虑: 具体是: 第一件事: “今天就抽空打个电话给你爸妈, 告诉他们你已经没事了, 具体不用多说, 汇 报结果就行。”让学生打电话给父母, 是主动避免父母再来关心过问可能再度 引发消极情绪的可能性, 只让学生汇报结果, 是为了避免复述详细的过程, 可 能导致消极情绪复燃。总的原则是在情绪转折点初期, 尽量减少负面刺激点, 切断失恋学生的消极情绪循环。第二件事: “抓紧去做最重要最紧急的事, 不 要让自己成为怨妇, 也不要再去找其他人倾诉, 因为如果别人不擅长劝导, 不 仅帮不上你的忙, 还可能火上浇油。”这一点非常重要, 前者是转移注意力, 由消极情绪的破坏性转向正事的建设性，后者是为了避免向不得当的对象倾诉 带来的二次伤害, 这种伤害可能是无心的,但无心之过同样能产生伤害的效果。 这样做的目的同样是为了切断失恋学生的消极话轮循环。第三、四件事详见 5 . 2.5 中的示例分析。

\subsection{3 策略三: 建立得当的心理参照系, 弱化极端悲观的自我判断}

以片段二对话中的 “我有几个学生都是正式谈了两年以上才分手了, 而且 有的都已经同居过了” 为例, 在当事学生感觉自己是世界上最不幸最倒霉的人 时, 这种自我极端悲观判断会产生晕轮效应, 并在消极情绪的漩浴中不断人为 放大。如果能及时为其建立起得当的心理参照系, 则可快速消解这种盲目消极 的恶性循环。因为, 当一个人知道自己所遭受的痛苦别人曾经或者正在遭受时, 就会立即获得一种心理平衡。不是心肠不好, 而是借助他人的类似痛苦甚至更 痛苦的经历, 消除自己是最不幸的错误认知, 在心理参照系中重新定位自己, 踩住自己消极情绪的刹车, 进入相对冷静客观的判断频道中。

值得注意的是，心理参照系的建立，最好以同一水平或更高水平的对象做 为参照对象, 而不能用更低水平的对象做为参照, 那样可能灭火不成, 反而会 火上浇油。就像文章开头 C 教授所举的例子, 因为参照对象是周恩来总理而有 效, 如果举的是一个既无社会影响又无经济实力又无其他成就的对象做为论据, 则效果可能会适得其反。

4.2.4 策略四: 引用切题应景的哲理故事警言潮语, 引发反省与共鸣 大学生不喜欢传统的说教, 尤其是在失恋的消极状态下, 更是对缺乏信息 
差和智慧含金量的说教话语极为排斥。此时失恋学生更希望听到哲理小语、警 言潮语或真实的类比小故事。如片断三中下划线部分的回应话语，正是此类话 语类型。比如 “谈恋爱就像剥洋苟, 总有一层会让你流泪。我爱你时, 你说什 么就是什么。我不爱你时, 你说你是什么。难免埋怨时间的手, 把相爱写成相 爱过。等待你的关心，等到我关上了心。“浪漫”是一袭美丽的晚礼服，但你 不能一天到晚都穿着它。所以，你必须找到除了爱情之外，能够使你用双脚坚 强站在大地上的东西。”

片断三:
Labour 12；07；27
他可能是真的另有想法或另有新欢，但不想刺激你，你也不 用去求证，好聚好散，两人相爱过，不成情侣，至少不能成 为敌人, 这是我一向的主张。
Labour 12；09；01
谈恋爱就像剥洋葱, 总有一层会让你流泪。我爱你时, 你 说什么就是什么。我不爱你时, 你说你是什么。难免埋怨 时间的手，把相爱写成相爱过。等待你的关心，等到我关 上了心。“浪漫”是一袭美丽的晚礼服, 但你不能一天到 晚都穿着它。所以, 你必须找到除了爱情之外, 能够使你 用双脚坚强站在大地上的东西。

Labour $12 ; 10 ; 21$

人生有时就像电脑, 说死机就死机, 没得商量。需要重启 时请不要犹豫。可以看出你非常乘，找几件有建设性的事 去做并做成，这样你就会重新找到自己的坐标。

终是迷惆 $\cdots 112 ; 11 ; 32$

好, 我会努力尝试的

Labour $12 ； 12 ； 28$

放弃自认为重要的东西比人们想象的要容易些, 困难在于 开始。一《如果在冬夜, 一个旅人》

一念心清静, 处处莲花开。

终是迷惆 $\cdots 112 ； 13 ; 52$

我和他还能当朋友吗

Labour 12; 15; 54

只要淡淡的就好, 不一定是朋友, 但至少不要是敌人

终是迷惆 $\cdots 12 １ 2 １ 8 ； 55$

我身边有他送的小东西, 看到的时候总会想起他, 难受。是 因为我没有放下才会这样吗? 那么, 我需要还给他还是怎么 
回应失恋大学生网络心理咨询 110
On the Rhetorical Strategies of Psychological Discourse in

E-Counselling Response to Lovelorn College Students

处理?

国庆我没回去，他来了。送了一个贵重的包包。我没用。是 否应该还给他

Labour $12 ； 21 ； 57$

有机会就还，但不用特地去还，一下子什么都不想了也不正 常不现实, 慢慢淡然就行。不久后的一天你将破蛹而出, 成 长得比人们期待的还要美丽, 但这个过程会很痛, 会很辛苦, 有时候还会觉得灰心。面对着汹涌而来的现实我们可能会觉 得自己渺小无力。但这，也是生命的一部分。做好现在我们 能做的应该做的建设性的事，功夫不负有心人，以物理时 间换心理空间，一切都会好起来的。

Labour 12；24；26

多年以后, 我们都长大了。经过谎言, 承受欺骗, 习惯敷 衍, 忘记誓言, 放下了一切。世界惩罚了我们的天真, 磨 损了我们的梦。但内心还是不断地闭合, 勇敢地开放, 一 往无前地爱。既然无法得到, 索性就放手成长吧。年少的 忧伤是人生必经的花园。一潘云贵《我们永远都是年轻 的模样》

Labour 12；25；47

生命中总会有无数个擦肩而过，不是每个相遇都能凝结成 相守, 不是每个相邀都能转化成相知。一辈子那么长, 生 活中变数那么多, 有时你以为会永远陪你走下去的那个人, 居然只能陪你一段路。幸好我们总会保有一点对于永远的 奢望, 不至于错过下一次爱情来的时候。

终是迷惆 $\cdots 12 １ 2 ２ 8 ； 29$

我怕自己再也不敢相信爱情

Labour 12; 31; 16

Keep calm in favourable circumstances. Keep composed in adverse situation. 不管外界如何喧器，知道自己要做什 么就行了。发展才是硬道理, 谈恋爱的条件, 就是让自己 从精神到物质，从灵魂到肉体，因为有了对方都比从前的 状态更好。否则何必呢? 就算你已经为他投入了很多时间 甚至金钱，该离开的时候也要利索点离开。勇于承担恋爱 的沉没成本, 是展开新生活的前提。至于你的怕不再敢相 信爱情的担心，也不是你的专利，刚分手的人通常都会这 样想，我不是说了吗? 以物理时间换心理空间，时过境迁， 就会水到渠成。 


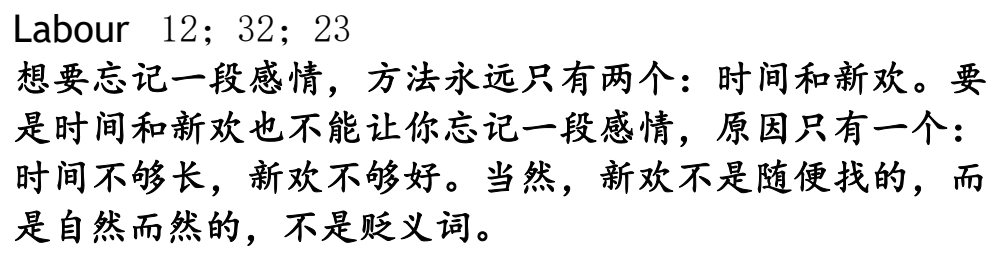

警言潮语的应用，体现在时间维度的张力、空间维度的张力、虚实相间的 张力、互文维度的张力和心理维度的张力, 触及咨询者原有的知识结构, 调动 其共享信息, 易引起失恋学生的共鸣与反省, 以 “他山之石, 可以攻玉” 的曲 线张力, 激发其同理心, 消除其心理壁垒和失恋阴影。例如, 有一位大学生抱 怨自己找的男朋友不够理想，笔者简要回复如下:

在正确的时间遇上正确的人，那是童话; 在错误的时间遇上正确的人或在 正确的时间遇上错误的人, 那才是青春。结婚之前老觉得适合自己的伴侣很少, 结婚之后发现适合自己的伴侣其实很多。但别忘了，大成若缺，没有什么是完 美的。女娲要辛苦折腾五彩石补天, 为什么? 唐僧西天取经, 经书泡水还缺角 了呢。生活要有点裂缝, 阳光才照得进来。

“在正确的时间遇上正确的人，那是童话; 在错误的时间遇上正确的人或 在正确的时间遇上错误的人，那才是青春。” “大成若缺，生活要有点裂缝， 阳光才照得进来。”这两句话就是典型的哲理潮语。“女娲补天” 和 “唐僧取 经” 这两个典故的类比, 也成为言简意赅的生动论据。在回应失恋学生的心理 咨询时, 熟记并得体应用这些哲理潮语和生动典故, 可以起到事半功倍的话语 
回应失恋大学生网络心理咨询 的话语修辞策略研究
On the Rhetorical Strategies of Psychological Discourse in

E-Counselling Response to Lovelorn College Students

修辞效果。

4.2.5 策略五: 提供重启心灵的具体化建议, 引导学生投身建设性事务

在回应另一失恋大四学生心理咨询的案例中, 学生面临专业八级考试备考、 学位论文写作、应聘面试三件重要的事情, 在初步消除其失恋的极端消极悲观 情绪并确认其放弃轻生念头后, 笔者进一步对这三件重要的大事一一进行针对 性很强的个性化指导，这一点正是很多心理咨询师无法完成的。只有提供重启 心灵的具体化专业建议，成功引导失恋学生投身建设性事务，才更有可能以正 能量的建设性巩固初步扭转的消极情绪。笔者花了半小时引导这位大四学生放 弃轻生念头, 又花了一小时指导其先备考专业八级考试和抓紧学位论文写作, 提供系列备考资源和论文写作要点范本，并承诺在后续应聘面试时继续指导。 两个月后, 这位学生获得专业八级考试优秀的成绩, 学位论文也顺利通过答辩, 并在激烈的竞争中脱颖而出, 进入一所县城重点中学任教。

在本例中, 如 5.2.2 中所述, 笔者在心理咨询对话的结尾, 叮嘱这位大四 失恋学生去完成四件事:

第一件是 “今天就抽空打个电话给你爸妈, 告诉他们你已经没事了, 具体 不用多说，汇报结果就行。” 第二件是 “抓紧去做最重要最紧急的事，不要让 自己成为怨妇, 也不要再去找其他人倾诉, 因为如果别人不擅长劝导, 不仅帮 不上你的忙, 还可能火上浇油。” 这两件事均是为了切断失恋学生的消极话轮 循环。

第三件是 “记录每天做重要正事的细目表, 在每件正事之后写上所投入的 分钟数。” 第四件是 “你有写微博记日记的习惯, 从今天起, 每天写三件值得 开心的事，大小不限，坚持 21 天。” 这两件事都是通过具体化的正事记录， 将正能量视觉化, 形成建设性的良性循环, 并在完成正事的过程中不断积累成 功与进步的喜悦, 有利于进一步压缩消极情绪与消极话轮拓展的空间, 更快地 修复消极情绪与内心伤害。

孔子曾称自己 “发愤忘食, 乐以忘忧” , 浸淫于学习与工作可以催人乐观 向上，而饱食终日无所事事的结果很可能是无事生非。“无事生非” 意指 “无 缘无故找岔子，存心制造麻烦”，此处不妨趣解之，即：无事可做，就容易惹 出是非。一个人如果没有建设性正事可做, 就算是正常人都容易生出是非来, 更不用说是一位处于失恋状态的大学生了。所以, 引导学生投入建设性事务, 将其注意力从消极循环中转移出来, 是走出失恋困境并实现可持续发展的关键 一环。

\subsection{6 策略六: 借力互动平台群体话语, 强化多管齐下的劝导实效}

笔者将具有代表性的 $\mathrm{QQ}$ 心理咨询对话内容, 隐去个人信息和敏感内容, 并在征得咨询学生同意后，发布在笔者的 $\mathrm{QQ}$ 空间日志中，吸引数以万计的访 客浏览, 并留下几百条的反馈感悟, 这些话语互文反馈具备很强的话语张力, 
具有如下三个功能: 一是可以助力强化对原匿名咨询者的劝导实效, 二是可以 进一步建立和巩固原匿名咨询者的心理参照系，三是可以以 “他山之石，可以 攻玉”的思路,给那些也存在类似困惑却无缘得到心理咨询辅导的其他访客(包 括大学生）以 “无心插柳柳成荫” 的帮助。不论是学生、老师、家长, 亦或曾 经的师生、未来的家长, 留言反馈均易引起共鸣与反思, 并在其后续的引用和 再引用过程中进一步强化甚至倍增其辐射扩展的正向效应。

精选部分 QQ 空间日志的学生留言回复, 篇幅所限, 详细分析不再展开。

7 楼 评论时间：2010-08-15 02:13:52

毛老师, 谢谢您解开了我最近的困惑, 我不能当逃兵, 要把不想做的事情 也做好, 不能因为心情不好就自我葄落, 爱情是人生的要素, 但不是人生的全 部。

8 楼 评论时间：2010-08-15 02:53:11

“放射自己的光，无须吹灭别人的灯。”"人对了，世界也就对了，观念对 了, 行动也就对了。” “工作不是你想干什么就千什么, 而是有能力把不想千 的事情做好。”

一语点醒梦中人，醍醐灌顶，也解开了我最近的心结，谢谢毛老师!

24 楼 评论时间：2010-08-20 11:02:27

毛老师对学生 (无论是不是您自己的学生) 确实很用心, 学习的好榜样。 不知道的人, 还以为您是心理学专业的。我们做辅导员的, 更要向毛老师多学 习! 您不仅帮了学生, 其实也无意间帮到了我。您的话很有内涵很正能量且很 有说服力, 尤其是遇上挫折时的调适与应对之道。谢谢您分享的这些答疑对话, 让我知道一些我从来都没有想过的问题和角度。不仅让我受益，还让我身边很 多好友和学生受益!

32 楼 评论时间: 2010-08-28 09:36:11

听君一番话, 胜读十年书! 好几句都值得我们尝试! 看同学经历, 感同身 受, 听毛老师的话, 醍醐灌顶, 豁然开朗! 路漫漫其修远兮, 吾将上下而求索! 闲暇时光, 进来走走, 总能受益匪浅一番。就像久旱逢甘霖, 润泽心田, 洗涤 混世尘埃。纵使书海无边, 或许只有似曾亲切的言语, 才更为意味深长。需静 静回味，慢慢咀嚼，心中困惑，尽皆释然。

33 楼 评论时间：2010-09-08 06:01:55

在混乱中微笑着前进。说的真好! 受益匪浅! 毛老师虽然不是专门开导我 的, 但效果是一样的, 我终于想通了! 不愿意承受爱情沉没的成本, 是一定会 变成怨妇的。我不想再当怨妇了, 听您的, 在混乱中微笑着前进! 您说的对: 真正的强者，是那些能够含泪奔跑的人！衷心谢谢毛老师！

52 楼 评论时间：2013-08-07 03:03:10

虽然我奔 4 年龄的人, 每次来毛老师的空间都很受益。每一句大爱大智慧 
回应失恋大学生网络心理咨询 的话语修辞策略研究
On the Rhetorical Strategies of Psychological Discourse in

E-Counselling Response to Lovelorn College Students

都意味深长，充溢着诗意，蕴含着哲理，无意间解开了我 $\mathrm{N}$ 多心结！关键是： 深入浅出, 非常接地气, 不是大话套话连篇。要是我当年能得到您这样的指点, 哪怕是这种网上的间接指点, 相信当年自己在感情挫折时, 可以少走很多弯路。 我让我孩子来读您的文章, 他也感觉受益匪浅。

这些在线互动留言曲线救国,往往能起到“无心插柳柳成荫”的共鸣效果。 数以百计的访客反馈, 自然建立起群体心理参照系, 不仅对原匿名咨询者具有 正向效应（原咨询者也能看到这些留言），也对其他访客以共鸣与反思，其效 应也可能进一步得以辐射扩展, 从而借力互动平台群体话语, 强化多管齐下的 劝导实效。

\section{5. 结语}

失恋大学生日益倾向于向自己信任的教师寻求网络心理咨询帮助, 大学生 网络心理咨询应用前景日益广阔。本研究将网络心理咨询分为 Email 延时话语 和在线 $\mathrm{QQ}$ 即时话语，通过调查访谈分析，指出其与传统面询相比所具备的优 势, 即远程便捷性、匿名私密性、资质安全性。通过案例分析，研究发现：回 应失恋大学生网络心理咨询的话语修辞策略主要有：A）巧设环环相扣的引导 性问题，了解失恋学生的真实困扰盲点; B）运用切中要害的适时反问，切断 失恋学生的消极话轮循环；C）建立得当的心理参照系，弱化失恋学生极端悲 观的自我判断；D）引用切题应景的哲理故事警言潮语，引发失恋学生的反省 与共鸣；E）提供重启心灵的具体化建议，引导失恋学生投身建设性事务；F） 借力互动平台群体话语, 强化多管齐下的劝导实效。

目前网络心理咨询的研究不足之处主要有五个方面:（1）网络心理咨询 在应用方面的研究尚未建立合理的心理健康服务标准; (2) 目前的网络心理 咨询研究样本大多缺乏代表性; (3) 心理咨询师通过在线与咨询者建立联系, 移情和反移情研究尚未引起足够重视; (4) 评估在线关系需要新的量表和技 术, 但如何将这些变量可操作化还需要研究; (5) 关于电脑媒介沟通的研究 还应考虑一些特殊问题，如：伦理、技术、逻辑、上网经验差异、缺乏非语言 线索等问题。

总之，网络心理咨询研究难度不小，但具有开创意义和广阔的应用前景。 大学生心理咨询已成为全社会关注的焦点, 对大学生的成长具有重要意义, 具 有广阔的研究和发展空间。网络心理咨询研究的进一步细化、深化，将进一步 推动心理咨询多领域的协同发展。展望网络心理咨询的研究, 主要有如下三个 方面:

第一，研究视角多元化。随着现代虚拟信息技术的不断发展，人机界面的 不断改进, 网络心理咨询也将更加技术化、科学化、人性化, 逐步减少网络带 来的负面影响。网络心理咨询研究不应局限在文本上的心理咨询内容研究, 而 应进一步拓展到网络视频咨询中的口头互动、语音交流以及多模态互动交流研 究领域。 
第二, 研究理论系统化。网络心理咨询研究的理论基础应在借鉴传统的面 对面心理咨询或国外相关理论与实践的基础上, 更加注重跨学科融合（如: 社 会语言学、神经语言学、语料库语言学、教育心理学、修辞学等），探索建构 植根于本土、符合中国国情又适合研究问题的理论体系。

第三，研究方法前沿化。从相关学科中借鉴适合心理咨询研究的国际前沿 新方法和新视角（如：语料库分析方法、ERPs 和 fMRI 等），进一步推动心理 咨询研究的精细化和科学化。心理咨询研究将在兼顾定量和定性分析方法的基 础上, 从注重典型案例文本分析转向大数据分析、多模态分析和科学实验分析。

作者注: 郑凌茜博士、吴丹苹博士、张妍博士、张薇博士、孙钦美博士、 高丽珍同学、林丽风老师、黄春蕾老师、李慧敏老师、李兴忠老师、唐昕韵同 学、程璐璐老师、刘叶红老师、林素琴老师、何文涁同学对论文进行了多轮细 致的修订, 谨此一并致谢。

\section{参考文献}

Brown, C., 2012. Online Counselling: Attitudes and Potential Utilization by College Student. The Faculty of Humboldt State University.

Dunn, K., 2012. A Qualitative Investigation into the Online Counselling Relationship: To Meet or not to Meet, That is the Question. Counselling and Psychotherapy Research 12, 4: 316-326.

Glasheen, K. J., I. Shochet \& M. A. Campbell, 2015. Online Counselling in Secondary Schools: Would Students Seek Help by This Medium? British Journal of Guidance \& Counselling 1, 2: 1-15.

Glueckauf, R. L., 1993. Improving Assessment in Rehabilitation and Health. London: SAGE Publications.

Grohol, J., 1995, 2012, 2015. The Future of People with Severe Mental Illness Is Safe. http://www.huffingtonpost.com/dr-john-grohol/ and http://www.grohol.com/.

Johansson, A., E. Brunnberg \& C. Eriksson, 2007. Adolescent Girls' and Boys' Perceptions of Mental Health. Journal of Youth Studies 10: 183-202.

Maclean, A., K. Hunt \& H. Sweeting, 2013. Symptoms of Mental Health Problems: Children's and Adolescents' Understandings and Implications for Gender Differences in Help Seeking. Children \& Society 27: 161-173. 
National Board of Certified Counsellors, 2001. The Practice of Internet Counselling. Retrieved December 21, 2008, from http://www.nbcc. org/ethics/Default.aspx.

Pollock, S. L., 2006. Internet Counselling and its Feasibility for Marriage and Family Counselling. The Family Journal 14, 1: 65-70.

Smith, C. L., 2012. Mental Health and Help-seeking in Adolescence. Australasian Epidemiologist 19, 1: 5-8.

Tanrikulu, I., 2009. Counsellors-in-training Students' Attitudes Towards Online Counselling. Procedia Social and Behavioural Sciences 1: 786.

Wehrman, J. D., 2004. A Survey of CACREP Liaisons Attitudes Regarding the Ethics, Training, and Practice of Internet Counselling in Counsellor Education Programs. Division of Counselling and Psychology in Education of University of South, Dakota.

邱芬，2008，我国高校网络心理咨询的现状及对策研究 [D]。南昌大学硕士论 文。

宋大伟, 2008, 网络心理咨询的局限与应用对策研究 $[J]$ 。河南师范大学学报 （哲学社会科学版），(5)：221-223。

晏小萍, 2008, 心理咨询话语结构分析：控权与赋权 $[\mathrm{J}]$ 。语言文字应用，(3)： $63-71$.

张芳、毛浩然, 2008, 大学生心理咨询现状与对策 $[J]$ 。河北北方学院学报，(3)： $78-81$ 。 